\title{
A meteorological and photometric study of the Oukaimeden site
}

\section{Comparison with the Observatorio del Roque de los Muchachos using Carlsberg Automatic Meridian Circle data}

\author{
A. Jabiri ${ }^{1}$, Z. Benkhaldoun ${ }^{1}$, J. Vernin ${ }^{2}$, and C. Muñoz-Tuñón ${ }^{3}$ \\ 1 Laboratoire de Physique des Hautes Énergies et Astrophysique, Département de Physique, Faculté des Sciences Semlalia, \\ BP. 2390, Marrakech, Maroc \\ 2 Département d'Astrophysique, CNRS UMR 6525, Université des Sciences, F-06108 Nice Cedex 2, France \\ 3 Instituto de Astrofísica de Canarias, E-38200 La Laguna, Tenerife, Spain
}

Received February 28; accepted July 4, 2000

\begin{abstract}
In this paper, we present meteorological and photometric studies of the Oukaimeden astronomical site in Morocco. The meteorological measurements were obtained over 537 days. The photometric data were measured by both FIP and GONG instruments over 1182 days. We have also analysed the weather parameters at the Observatorio del Roque de los Muchachos (ORM) in the Canary Islands. More than ten years of measurements with the Carlsberg Automatic Meridian Circle (CAMC) are included, corresponding to 2931 nights of atmospheric extinction measurements.

These different data sets demonstrate photometric sky quality, wind speed and direction, local relative humidity, temperature and other parameters, such as the barometric pressure at the ORM and the precipitation at Oukaimeden. We have separated the day- and night-time data to provide information relevant to solar and stellar observations. Data from the Oukaimeden site have been compared to the ORM site, recognized as one of the best astronomical sites in the world and the results from our data confirm that Oukaimeden constitutes an excellent potential site for astronomical observations.
\end{abstract}

Key words: site testing

\section{Introduction}

Oukaimeden is a potential site for an astronomical observatory in Morocco $^{1}$. Precise astronomical

Send offprint requests to: A. Jabiri

1 The observing station of Oukaimeden is supported by Cadi Ayyad University and National Center for Coordination and Planning of Scientific and Technical Research. site-testing first requires a thorough preliminary study. The installation of astronomical equipment by groups such as IRIS (International Research of the Interior of the Sun), GONG (Global Oscillation Network Group) and FIP (Flux Integration Photometer) form the body of our meteorological database. We have selected the Canarian Observatories (Fig. 1) as an obvious comparison site with Oukaimeden because the Observatorio del Roque de los Muchachos (ORM) has been proven to be excellent for night-time observations (see, for example, McInnes \& Walker 1974; McInnes 1981; Brandt \& Wohl 1982; Murdin 1985; Mahoney et al. 1998; Muñoz-Tuñón et al. 1998). Assuming that both sites are driven by similar meteorological trends, we believe that Oukaimeden may also be a good candidate for astronomical research.

Both French and Moroccan researchers have selected Oukaimeden as an astronomical observing site. Benkhaldoun (1994) has already presented the criteria for this choice; therefore, for the purpose of this paper, we will simply summarize the relevant points.

The first search for a Moroccan observatory site began with data analysis taken from the Moroccan National Center of Meteorology (Kadiri 1983). This climatological and geographical preliminary investigation limited the selection to mountains located in the northern and southern regions. The northern area revealed good logistic conditions but poor photometric data. In the south, logistical and meteorological reasons led us to exclude some areas. Finally, Oukaimeden located in the high Atlas Mountains, (longitude: $7^{\circ} 52^{\prime} 52^{\prime \prime} \mathrm{W}$, latitude: $31^{\circ} 12^{\prime} 32^{\prime \prime} \mathrm{N}$ ) and $2700 \mathrm{~m}$ above mean sea level, was pre-selected as a potentially excellent site for astronomical observations.

Oukaimeden dominates the plain of Marrakech (the nearest city), and is located on a peak overlooking a village also bearing the name Oukaimeden. To the south and 


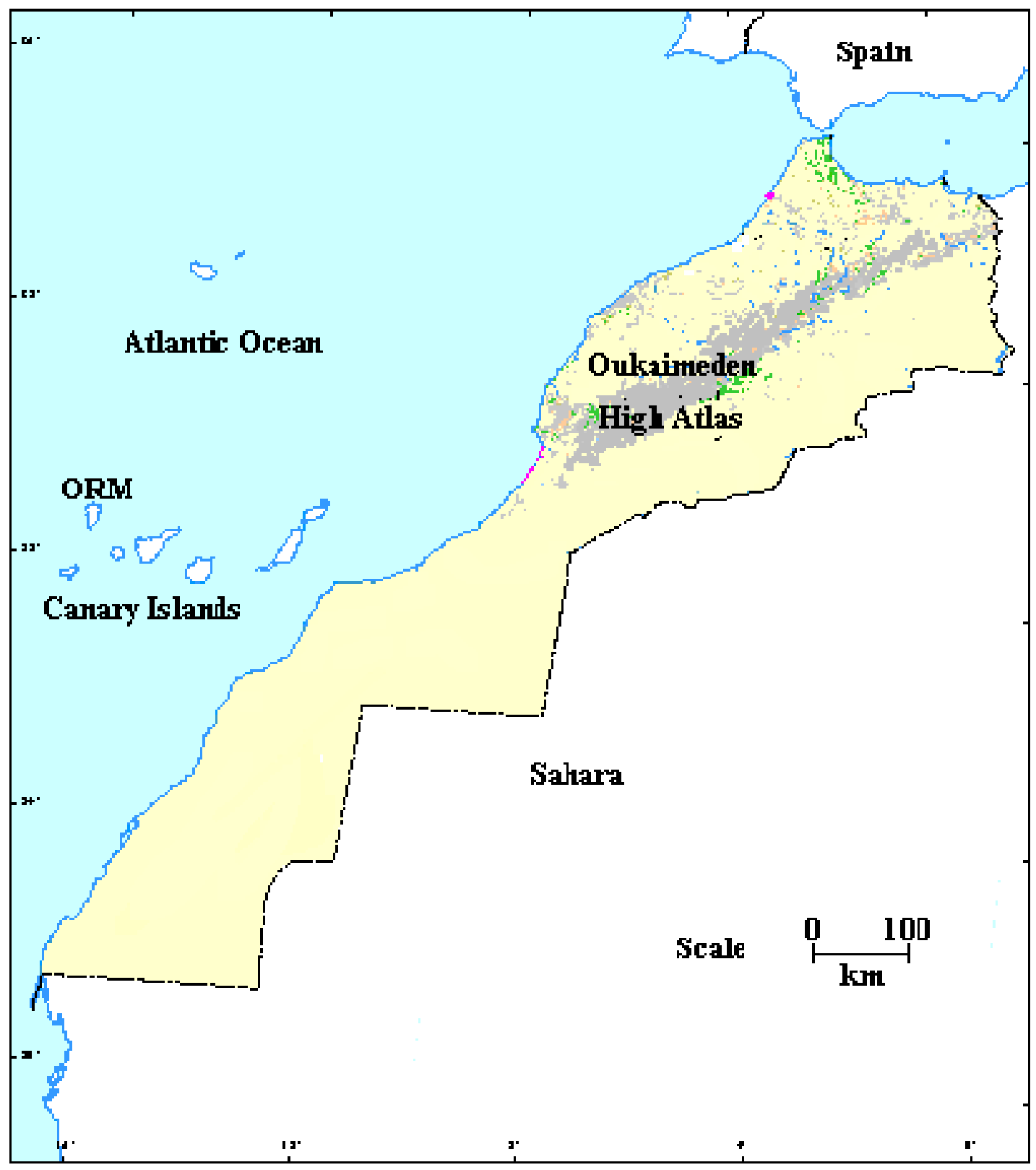

Fig. 1. Oukaimeden and ORM site localization

south-east, the $4000 \mathrm{~m}$ summits act as a natural barrier against wind-borne dust from the nearby Sahara Desert. Therefore, Oukaimeden is the first natural obstacle that homogeneous, unperturbed air masses meet as they arrive from the Atlantic Ocean. This particularly advantageous situation gives Oukaimeden the benefit of extremely transparent skies and enables us to predict the low atmospheric turbulence that characterizes coastal sites (Benkhaldoun 1994).

Weather conditions are so favourable at Oukaimeden that several groups studying helioseismology have also proposed the installation and development of international observation networks there. Currently The IRIS network observing the full solar disc is installed at the site.

The ORM, on La Palma (Canary Islands) has also been extensively studied. For a precise location and general surface layer meteorology see (Muñoz-Tunón et al. 1997; Mahoney et al. 1997, and references therein). In 1984, more than 14 European countries recognized the excellence of the Canary Island location and after signing formal agreements, set up the present ORM on an international basis. The $10 \mathrm{~m}$ segmented Gran Telescopio 
Canarias (GTC) is now being constructed at ORM, thereby increasing the Observatory's technological capabilities as well as assuring ORM's future as a renowned international observatory. In 1985, a special issue of Vistas in Astronomy (Murdin 1985) was dedicated to the Canarian Observatories to coincide with ORM's inauguration. This issue highlighted the characteristics which make the ORM a prime astronomical location.

During the last few years, spectacular progress has been made in the study of certain parameters that define atmospheric quality for astronomical observations. There have also been major breakthroughs in adaptive optics, high-resolution observational techniques, the modelling of orographical influences and dome and building design. At a recent conference entitled Site Properties of the Canarian Observatories astronomers reviewed and compiled new results concerning site-testing at the Canarian Observatories. The proceedings of this conference appeared in a special issue of New Astronomy Reviews (Muñoz-Tuñón 1998).

In this paper, we will present the surface layer meteorology of the Oukaimeden site gathered over more than two years of measurements. The results are given in Sect. 3. Section 4 describes the surface layer meteorology of the ORM based on CAMC data and in Sect. 5, we compare the potential of the Oukaimeden site for astronomical observing with the ORM.

\section{Variables to be studied}

Astronomers know that the following set of well defined atmospheric conditions makes for excellent astronomical observations: cloud cover should be minimal, the frequency of unfavourable weather conditions such as cyclonic storms and thunderstorms should be very low, the air above the observatory site should be dry, the change in air temperature during the night should be minimal and atmospheric transparency should be high.

Relevant surface layer meteorological variables for site characterization are air temperature $(T)$, barometric pressure $(P)$, relative humidity $(\mathrm{RH})$ and wind velocity $(\vec{V}$, broken down into speed and direction). Other parameters, such as sky transparency and photometric conditions, are taken into account to define the astronomical suitability (see Ardeberg 1983; Murdin 1985; Mahoney et al. 1998).

- Air temperature

Large changes in air temperature during the night make it more difficult to minimize dome and mirror thermal perturbations, known as dome and mirror seeing. The surface layer, due to convection (calculated from ground-air temperature differences), is also expected to be less significant when the temperature remains stable during the night;

At the ORM, the analysis of temporal behaviour of night-time seeing shows no dependence of seeing on time. No variation of seeing has been detected that could be associated with the beginning or the end of the night (Muñoz-Tuñón et al. 1997), supporting the negligible value of the surface layer seeing as reported by Vernin \& Muñoz-Tuñón (1994);

- Relative humidity

Relative humidity at ground level provides a useful value in determining conditions in which astronomical observations can be made. Humidity higher than, say, 90\% (Murdin 1985) makes observing unfeasible. The optical surfaces become wet and can be damaged. The percentage of hours with humidity less than $90 \%$ sets an upper limit on observable time. However, it is important to mention that relative humidity is a local measurement which relates poorly to the water vapour column at the site; therefore, relative humidity by itself cannot determine the suitability of a site for infrared observations;

- Wind speed and direction

Wind speed and direction also constrain the operating of telescopes. While wind speed of, say, $15 \mathrm{~m} \mathrm{~s}^{-1}$ may be taken as a typical maximum safe operating value (Murdin 1985), the maximum operational wind speed will also depend on the angle between the orientation of the dome slit and the oncoming wind;

The overall climate of the Canary Islands is determined by the trade winds, which are $90 \%$ prevalent in the summer and $50 \%$ prevalent in the winter (see Font Tullot 1956), and which arrive from the NE. The prevailing wind vector veers with height, and at the level of the Observatories, well above the inversion layer, the trades arrive from the NW (although there is a seasonal variation since the Azores anticyclone, which, together with the Canary Current, drives the trade winds, migrates in the north-south direction inaccordance with the change in declination of the the Sun (see Mahoney et al. 1998);

Recently, a correlation has been found between poor image quality and southerly winds at ORM, although these do not reflect typical conditions at the ORM. Further study of this correlation between wind direction and seeing could be of interest for climatology research (Muñoz-Tuñón et al. 1998);

- Barometric pressure

The trade winds in the Canary Islands correspond to a general anticyclonic circulation and provides a mean pressure value (at ORM) higher than that theoretically expected for this altitude. The frequency with which this happens is an indirect indication of high atmospheric stability;

Large temporal gradients in pressure are frequently associated with changes from anticyclonic to frontal weather and possible deterioration in observing conditions (storms, strong winds, clouds, etc.);

- Atmospheric extinction

While atmospheric extinction is determined by atmospheric transparency, spatial and temporal variations 
Table 1. Effect of the temperature difference between two consecutive nights on the dome and mirror seeing

\begin{tabular}{lccc}
\hline & $\begin{array}{c}\Delta T \\
\left({ }^{\circ} \mathrm{C}\right)\end{array}$ & $\begin{array}{c}\text { Dome seeing } \\
(\operatorname{arcsec})\end{array}$ & $\begin{array}{c}\text { Mirror seeing } \\
(\operatorname{arcsec})\end{array}$ \\
\hline Mean & 1.72 & 0.19 & 0.77 \\
Max. & 9 & 1.4 & 5.6 \\
\hline
\end{tabular}

of this variable are related almost entirely to differences in the concentration of suspended particles. Since observatory sites are usually in dry, isolated areas, dust is the most common particle that affects transparency. The quantity of dust in the air depends on the altitude of the observing site and its proximity to a dust source (e.g. deserts) and prevailing wind. Even if an observatory site lies well above the inversion layer, dust can still have an important effect on atmospheric transparency;

For example, in the Canary Islands, when eastern winds occur above the inversion layer, Saharan dust is carried over the islands from the desert interior of North Africa, degrading atmospheric transparency. Most of the time, however, the air reaching the observatory sites of the Canaries has traversed thousands of kilometres of featureless ocean and will accordingly be free from suspended particles. Therefore, with regard to atmospheric transparency, isolated oceanic sites have a decided advantage over continental sites provided that oceanic sites lie above the inversion layer (Guerrero et al. 1998, 2000; Murdin 1985).

In Figs. 2-11 plots are shown for mean monthly air temperature, daily mean air temperature, monthly temperature difference between two consecutive nights, mean monthly relative humidity, cumulative frequency of relative humidity, daily relative humidity, night-time wind rose, daytime wind rose, mean monthly wind speed and cumulative frequency of windspeed, respectively. In each figure, the left-hand panel corresponds to Oukaimeden (OUK) and the right-hand panel to the Observatorio del Roque de los Muchachos (ORM). The recorded data from $10 \mathrm{UT}$ to $16 \mathrm{UT}$ were used to sample daytime conditions and from $22 \mathrm{UT}$ to $04 \mathrm{UT}$ to study night-time conditions and from $0 \mathrm{UT}$ to $24 \mathrm{UT}$ refer to daily results.

\section{Oukaimeden surface layer meteorology}

The meteorological measurements were recorded from 1990 December to 1993 March. A breakdown of the observing station occurred during the months of 1991 September and 1992 November. The automatic station consists of a data acquisition unit provided with several analogue channels connected to meteorological sensors. The parameters measured were wind speed and direction, relative humidity, air temperature and rainfall.
All parameters were averaged and, together with extreme values, recorded every hour.

\subsection{Air temperature}

The statistical results of the temperature are presented in Figs. 2-4. In Fig. 2, we display monthly all-day mean, minimum and maximum values of the temperature (solid lines). Daytime and night-time mean temperatures are plotted (dotted and dot-dashed lines, respectively). From our data, we can see a seasonal variation: the cold months are October to March and the hot months are April to September. The daily mean temperature is $6.47^{\circ} \mathrm{C}\left(1.9^{\circ} \mathrm{C}\right.$ in winter, $12.36{ }^{\circ} \mathrm{C}$ in summer). The absolute minimum recorded during the night in $1992 \mathrm{March}$ is $-12.9{ }^{\circ} \mathrm{C}$ and the absolute maximum recorded during the day in 1991 August is $25.6{ }^{\circ} \mathrm{C}$.

The daytime and night-time mean temperatures are $9.48{ }^{\circ} \mathrm{C}$ and $4.7{ }^{\circ} \mathrm{C}$, respectively. The daytime minimum is $-3.9{ }^{\circ} \mathrm{C}$ recorded in 1991 March and the night-time maximum is $17.5^{\circ} \mathrm{C}$ recorded in 1992 August.

The daily variation of the temperature at Oukaimeden is presented in Fig. 3. Note that during the night, the temperature decreases very slowly, due to thermal cooling.

The statistics in temperature difference for two consecutive nights are illustrated in Fig. 4. The mean temperature difference is $1.72^{\circ} \mathrm{C}$. The maximum difference is $9{ }^{\circ} \mathrm{C}$ recorded in March. The seasonal variation is also negligible and shows that the stability of the nightly temperature is considerable at both sites.

Following Racine et al. (1991), we have determined the dome ("D") and mirror ("M") contribution to the seeing by using:

$\epsilon_{\mathrm{D}} \simeq 0.1(\Delta T)^{6 / 5}(\operatorname{arcsec})$

$\epsilon_{\mathrm{M}} \simeq 0.4(\Delta T)^{6 / 5}(\operatorname{arcsec})$

where $\Delta T$ represents the temperature difference between the dome (or the mirror) and the external atmospheric temperature.

Because most observatories use air conditioning, the difference in temperature between two consecutive nights might determine the magnitude of the dome and telescope mirror seeing. Let us define this difference $\Delta T$ :

$\Delta T=\left\langle\left|T_{j}-T_{j+1}\right|\right\rangle$,

where $j$ is a given night.

Then $\Delta T$ is used in Eqs. (1) and (2). In Table 1, we present the effect of such temperature difference on dome and mirror seeing. The seeing variation is mainly produced by mirror seeing.

\subsection{Relative humidity}

In Fig. 5, we present the monthly variation of the daytime, night-time and all-day relative humidity. Here we 

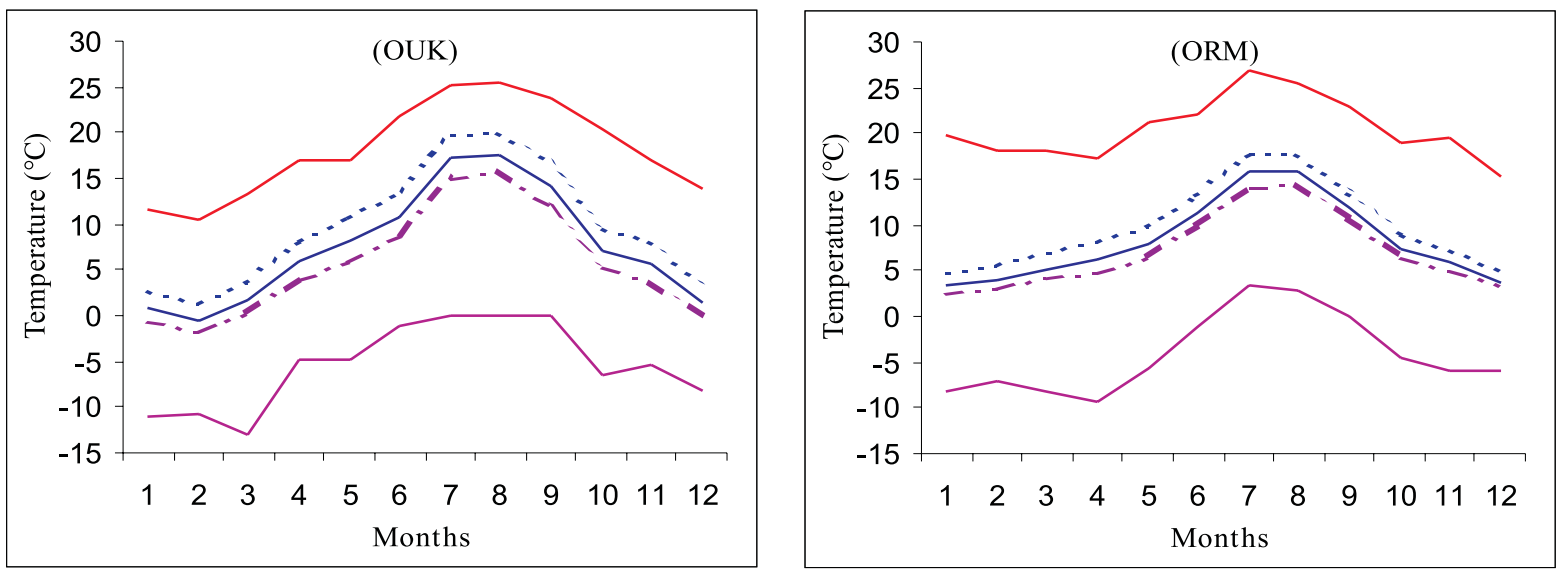

Fig. 2. Monthly variation of the temperature at Oukaimeden (left) and ORM (right). The mean variation is calculated by using respectively daily data (solid line), daytime data (upper dotted-line) and night-time data (lower dotted-dashed line). Upper and lower curves represent respective absolute maximum and minimum temperatures
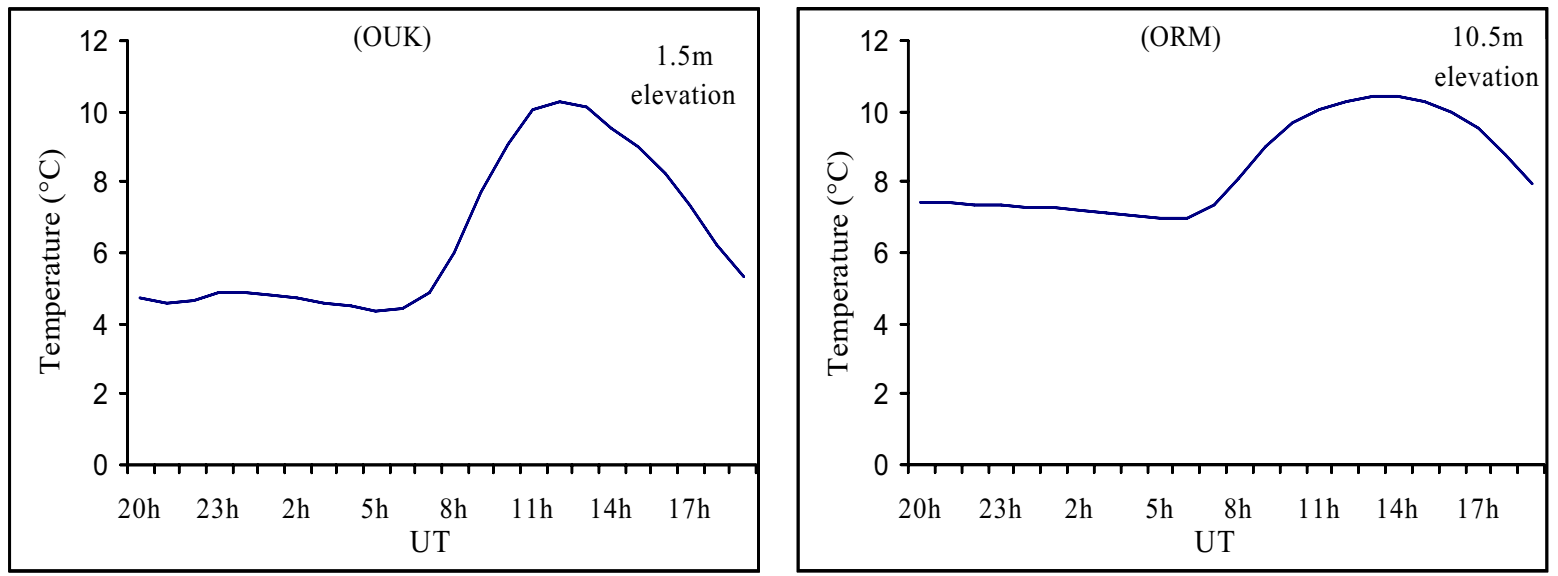

Fig. 3. Daily mean variation of temperature. Left: the temperature measured at $1.5 \mathrm{~m}$ above ground level at Oukaimeden. Right: the temperature measured at 10.5 above ground level at ORM
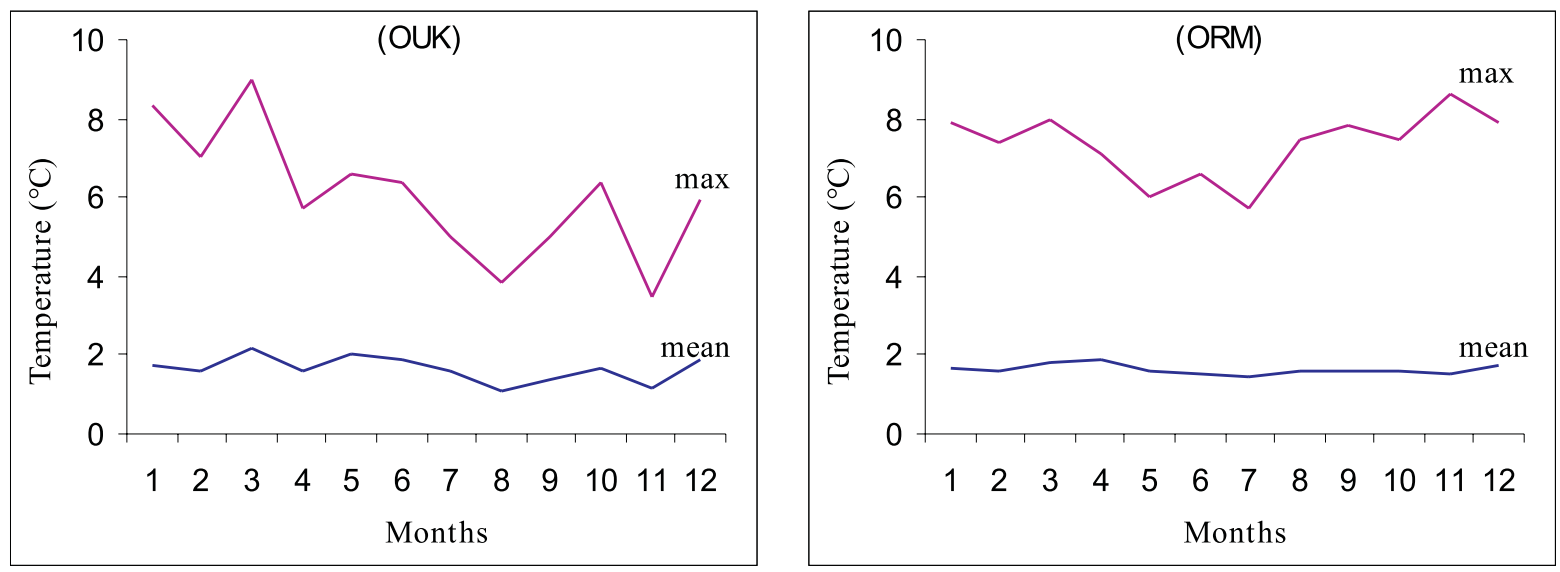

Fig. 4. Monthly temperature difference between two consecutive nights at Oukaimeden (left) and ORM (right). Data is calculated as the absolute difference of the mean night-time temperature from one night to the next 

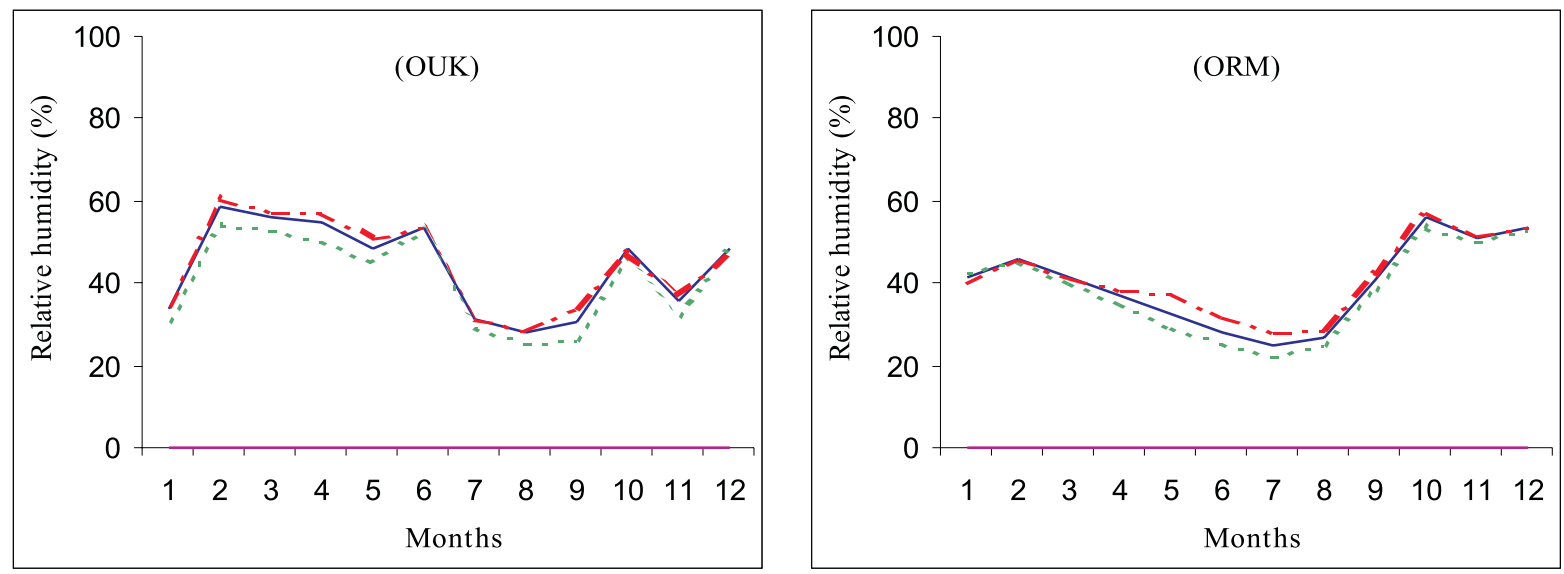

Fig. 5. Monthly variation of relative humidity at Oukaimeden (left) and ORM (right). Daily data is represented by a solid line, night-time data by dotted-dashed line and daytime data by a dotted line
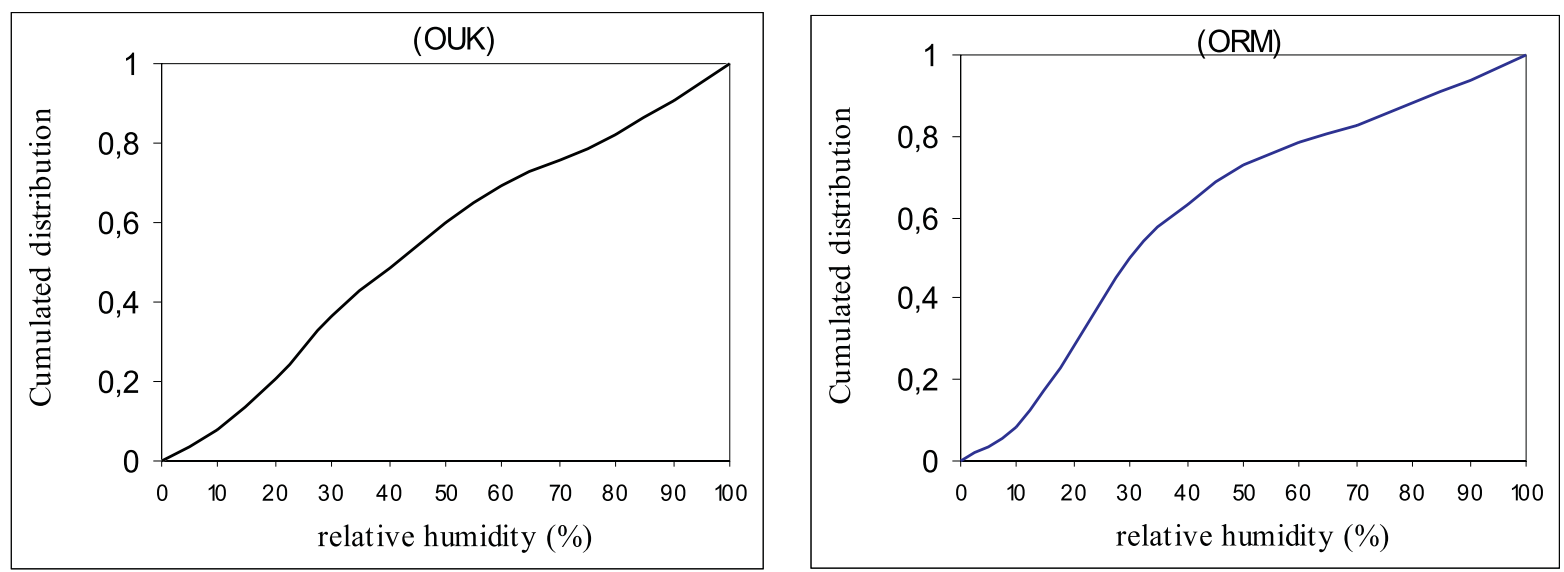

Fig. 6. Cumulative frequency of relative humidity at Oukaimeden (left) and ORM (right). The median value is $40 \%$ at Oukaimeden and $31 \%$ at ORM
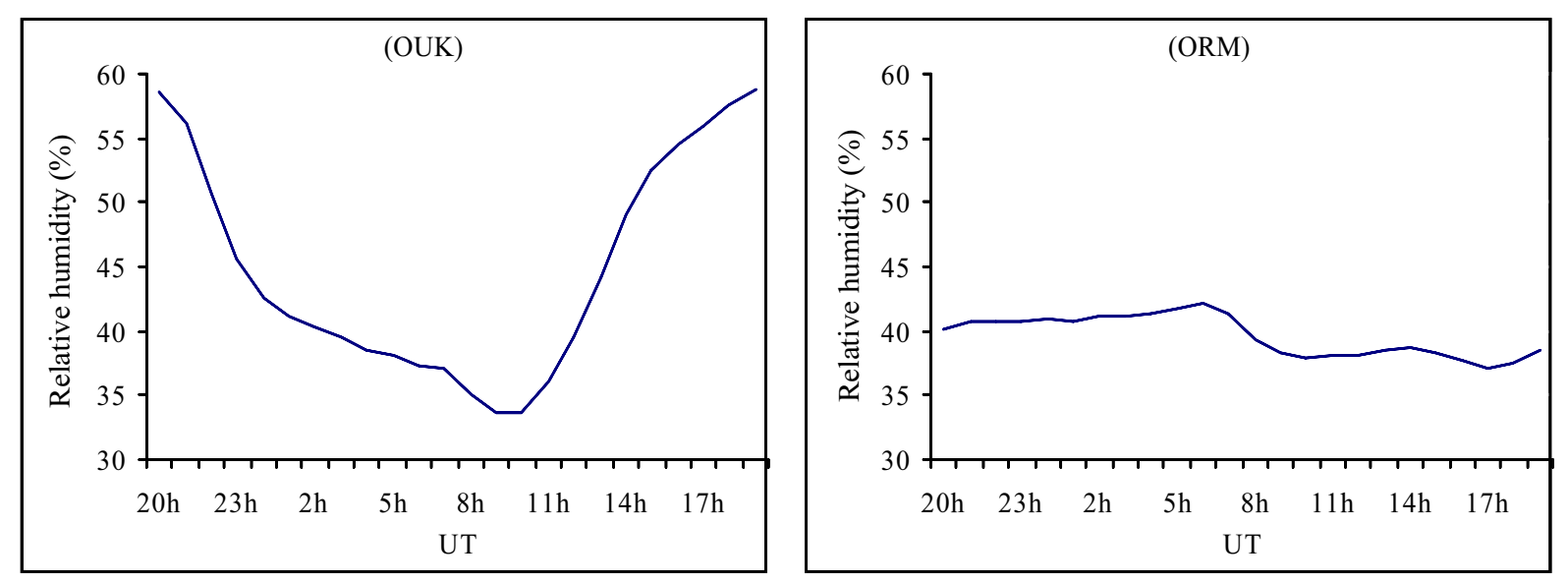

Fig. 7. Daily variation of relative humidity. Left: at Oukaimeden, the humidity decreases during the night until 10 am in the morning when it increases until the beginning of the evening. This variation is perceived by our photometric measurements. Right: at ORM, the stability of humidity is quite remarkable during the night and daytime 

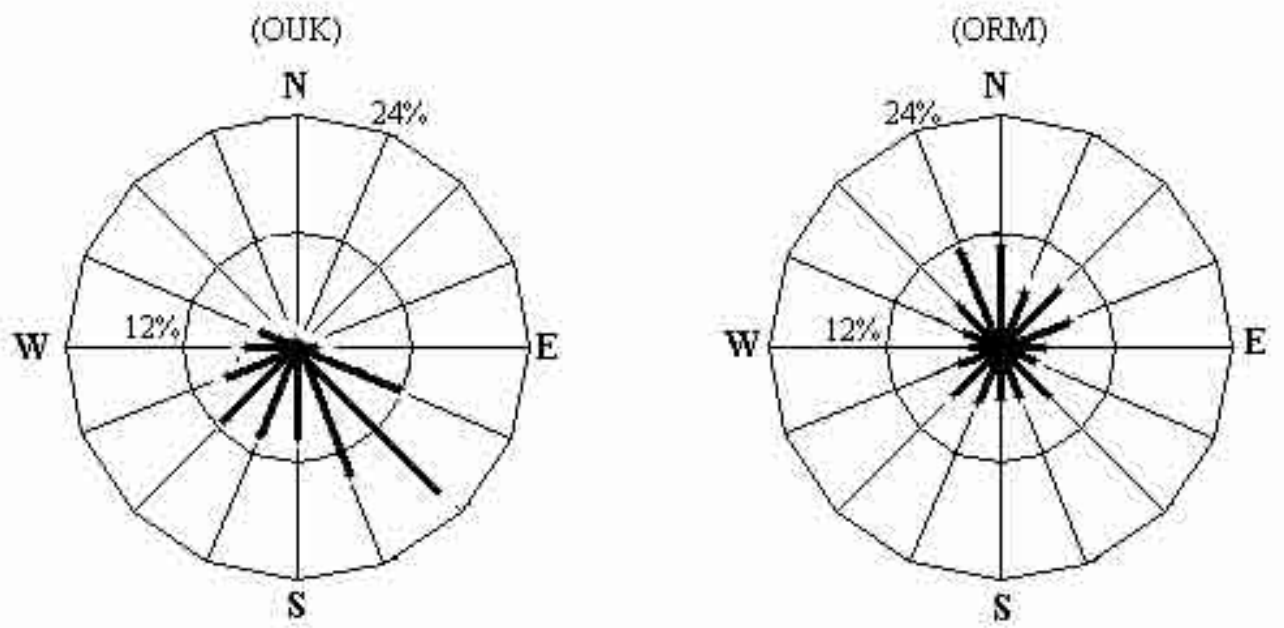

Fig. 8. Night-time windrose at Oukaimeden (left) and ORM (right). The frequency data is calculated respectively on 4541 hours of measurements at Oukaimeden and over 14748 hours at ORM
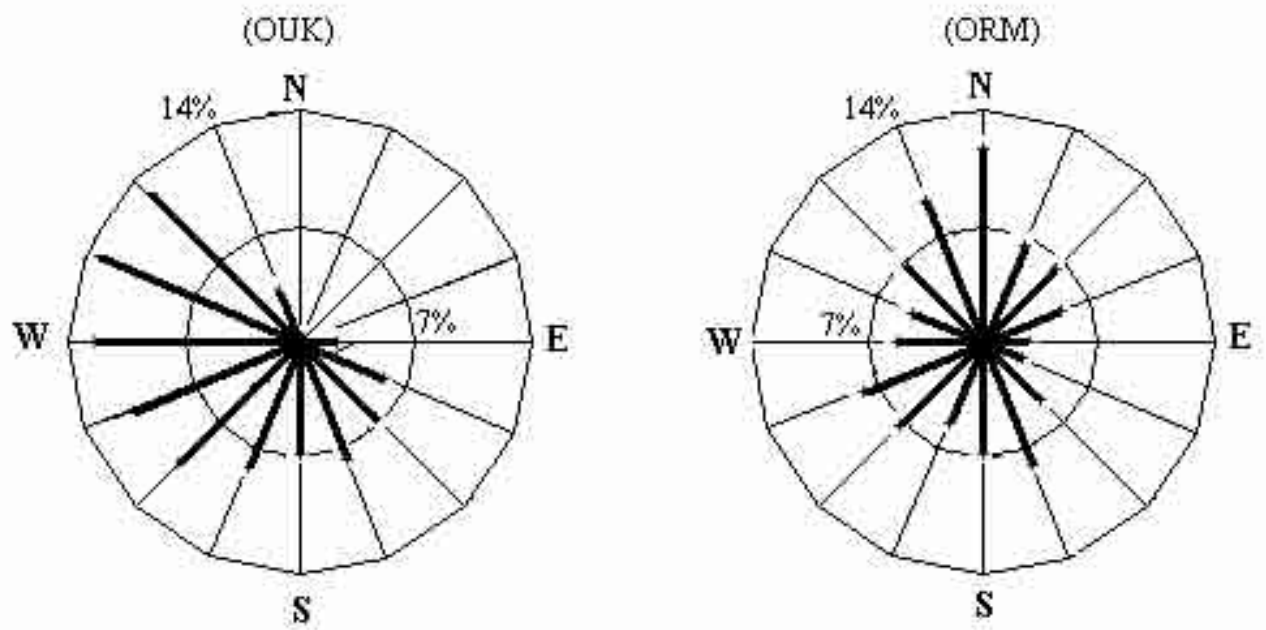

Fig. 9. Daytime windrose at Oukaimeden (left) and ORM (right). The frequency data presented is calculated respectively on 4650 hours of measurements at Oukaimeden and over 13575 hours at ORM
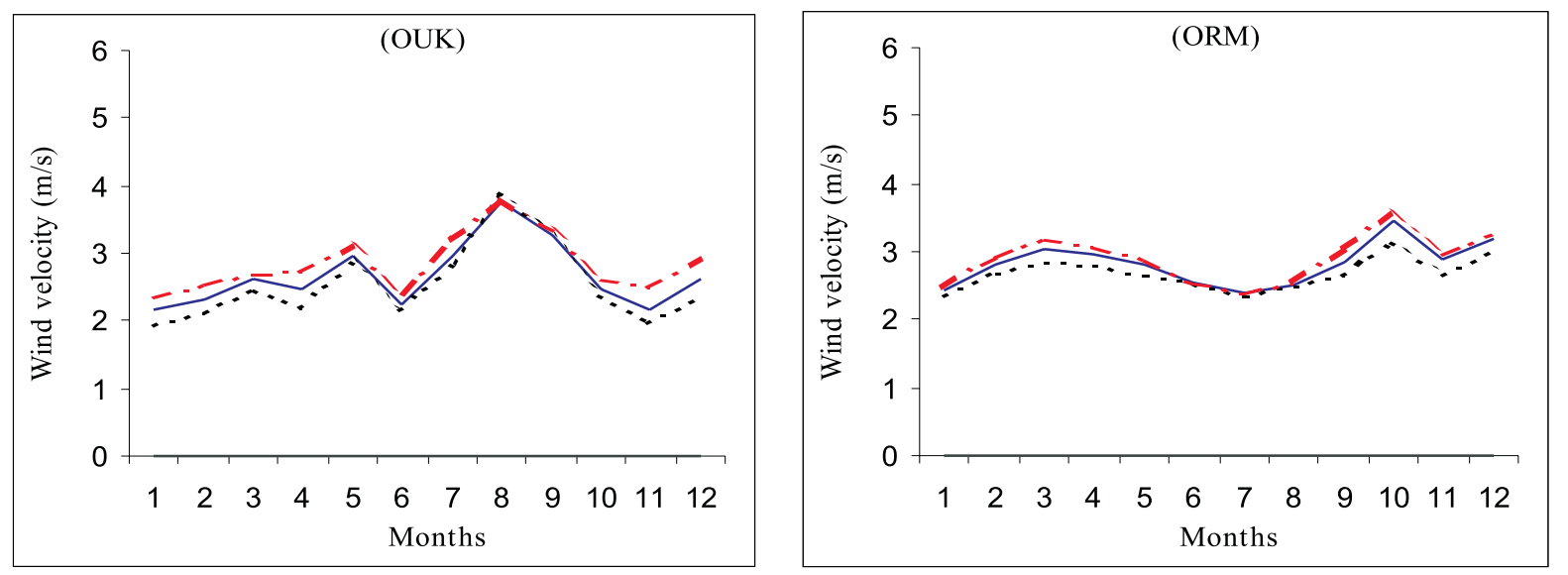

Fig. 10. Monthly variation of the wind velocity at Oukaimeden (left) and ORM (right). Daily data is represented by a solid line, night-time data by a dotted-dashed line and daytime data by a dotted line 

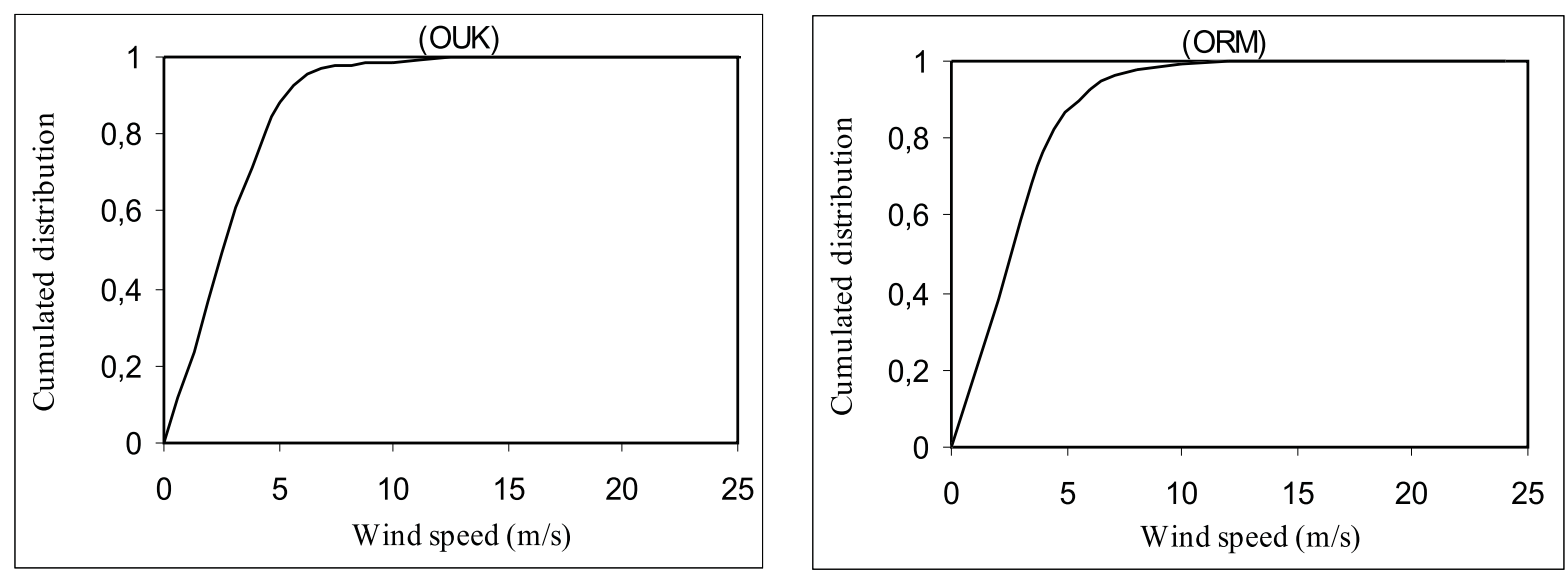

Fig. 11. Cumulative frequency of wind velocity at Oukaimeden (left) and ORM (right). The median value is $2.1 \mathrm{~m} \mathrm{~s}^{-1}$ at Oukaimeden and $2 \mathrm{~m} \mathrm{~s}^{-1}$ at ORM

Table 2. Night-time wind statistics $(4541 \mathrm{hr})$. Percentage of time per sector at Oukaimeden. The three wind speed intervals are respectively A: $[0,3$ [; B: $[3,6[$ [ C: $[6,+\infty[$

\begin{tabular}{|c|c|c|c|c|c|c|c|c|c|c|c|c|c|c|c|c|c|}
\hline & $\overline{\mathrm{N}}$ & $\overline{\mathrm{NNE}}$ & $\mathrm{NE}$ & ENE & $\mathrm{E}$ & ESE & SE & $\overline{\mathrm{SSE}}$ & $\mathrm{S}$ & $\overline{\text { SSW }}$ & $\overline{\text { SW }}$ & WSW & $\overline{\mathrm{W}}$ & $\overline{W N W}$ & NW & NNW & all \\
\hline$\overline{\mathrm{A}}$ & $\overline{0}$ & 0.1 & 0.3 & 0.5 & 1.4 & 5 & 13.4 & 7 & 5 & 5.6 & 8 & 6.9 & 5 & 3.7 & 1.9 & 0.7 & 64.5 \\
\hline B & 0 & 0 & 0 & 0 & 0.5 & 4.1 & 7.1 & 4.5 & 3.2 & 3.4 & 2.7 & 0.9 & 0.4 & 0.2 & 0 & 0 & 27 \\
\hline C & 0 & 0 & 0 & 0 & 0.1 & 2.3 & 1.3 & 2.7 & 1.2 & 0.8 & 0.1 & 0 & 0 & 0 & 0 & 0 & 8.5 \\
\hline & 0 & 0.1 & 0.3 & 0.5 & 2 & 11.4 & 21.8 & 14.2 & 9.4 & 9.8 & 10.8 & 7.8 & 5.4 & 3.9 & 1.9 & 0.7 & 100 \\
\hline
\end{tabular}

Table 3. Daytime wind statistics $(4650 \mathrm{hr})$. Percentage of time per sector at Oukaimeden

\begin{tabular}{|c|c|c|c|c|c|c|c|c|c|c|c|c|c|c|c|c|c|}
\hline & $\mathrm{N}$ & NNE & $\mathrm{NE}$ & ENE & $\mathrm{E}$ & ESE & SE & SSE & $\mathrm{S}$ & SSW & SW & WSW & W & WNW & NW & NNW & all \\
\hline $\mathrm{A}$ & 0 & 0 & 0.1 & 0.2 & 1.3 & 2.2 & 4 & 3.8 & 4.2 & 6.4 & 8.8 & 9.8 & 11.7 & 12.4 & 11.8 & 3.1 & 79.8 \\
\hline B & 0 & 0 & 0 & 0.1 & 0.9 & 2.2 & 1.6 & 1.4 & 1.6 & 1.4 & 1.6 & 1 & 0.7 & 0.8 & 0.8 & 0.4 & 14.5 \\
\hline $\mathrm{C}$ & 0 & 0 & 0 & 0 & 0 & 1.1 & 0.9 & 2.4 & 0.9 & 0.4 & 0 & 0 & 0 & 0 & 0 & 0 & 5.7 \\
\hline & 0 & 0 & 0.1 & 0.3 & 2.2 & 5.5 & 6.5 & 7.6 & 6.7 & 8.2 & 10.4 & 10.8 & 12.4 & 13.2 & 12.6 & 3.5 & 100 \\
\hline
\end{tabular}

also note a seasonal effect. The all-day mean humidity is $45 \%$. The daytime and night-time mean humidities are $46 \%$ and $42 \%$, respectively. The humid months span from February to June, whereas the driest ones are from July to January except October and December.

The cumulative frequency of relative humidity is shown in Fig. 6. The probability of high humidity is weak and given a $90 \%$ humidity threshold, only $8 \%$ of the observing time would be lost due to this cause.

The daily variation of relative humidity is shown in Fig. 7. It increases during the day with a peak at sunset and then drops rapidly at the beginning of the night. In our experience, this trend is supported by solar observation which shows us that atmospheric transparency is excellent in the morning yet decreases during the afternoon (Benkhaldoun 1993). Low humidity during the night also gives us favourable astronomical conditions.

\subsection{Wind speed and direction}

Wind statistics are presented in Tables 2 and 3 and Figs. $8-11$. The daytime and night-time wind readings are a compilation of a total of 9191 hours (Figs. 8 and 9). The highest wind speed frequency occurs within the A interval for both daytime (79.8\%) and night-time (64.5\%). At night this wind velocity interval peaks in the SE (21.8\%) and SW (10.8\%), whereas during the day we see a change in the wind pattern. The wind prevails from WNW direction $(13.2 \%)$. Within the $\mathrm{B}$ interval, the frequency distribution peaks at the night-time in the SE (7.1\%) and during the daytime in the $\operatorname{ESE}(2.2 \%)$.

The monthly variation of wind velocity is illustrated in Fig. 10. The data analysis shows a weak seasonal effect. The mean velocity is $2.63 \mathrm{~m} \mathrm{~s}^{-1}$. In all of our data, $60 \%$ of time, wind is included between 1 to $3 \mathrm{~m} \mathrm{~s}^{-1}$ and during $89 \%$ it is lower than $5 \mathrm{~m} \mathrm{~s}^{-1}$ (Fig. 11). Although the velocity is low enough, we found that the wind can reach a significant amplitude of $50 \mathrm{~m} \mathrm{~s}^{-1}$. We checked that a more than $25 \mathrm{~m} \mathrm{~s}^{-1}$ wind occurs within $5 \%$ of daily maxima and that a $50 \mathrm{~m} \mathrm{~s}^{-1}$ maximum occurs in 17 cases out of the 


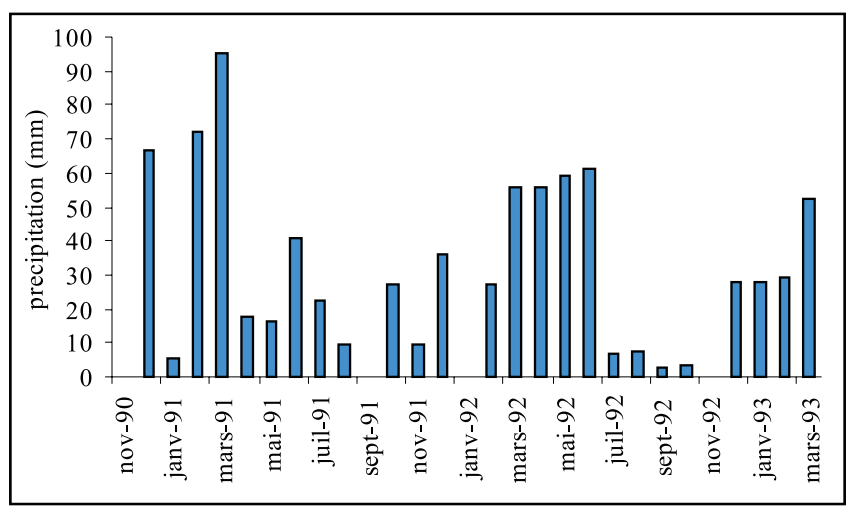

Fig. 12. Monthly total precipitation at Oukaimeden from November 1990 to March 1993

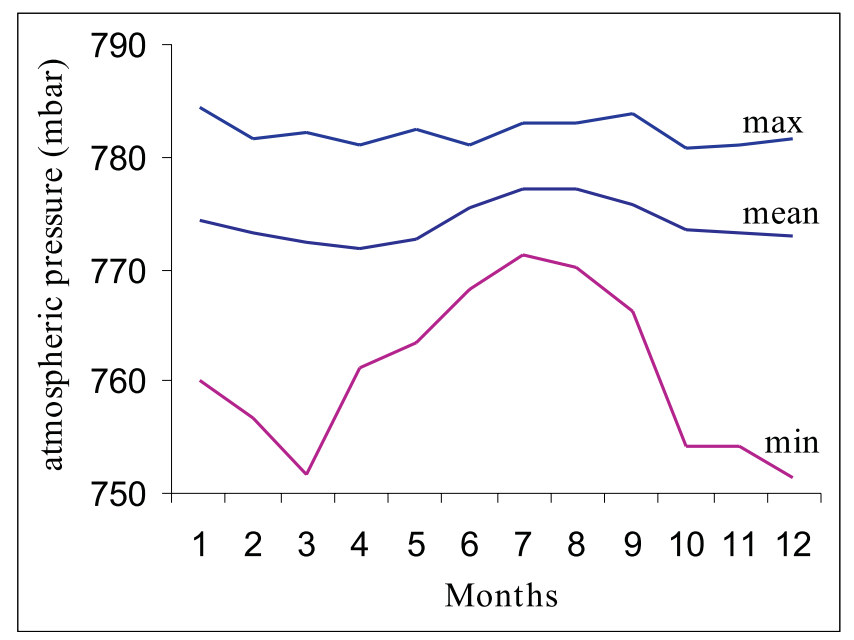

Fig. 13. Monthly variation of atmospheric pressure at ORM

652 daily maxima recorded. We have also analysed the wind velocity in terms of telescope management. Assuming a $15 \mathrm{~m} \mathrm{~s}^{-1}$ threshold (beyond which the telescope would cease operation) we lose only $0.2 \%$ of our research time, particularly in the winter.

\subsection{Precipitation}

The analysis of precipitation data allows us to establish that the annual mean at Oukaimeden is about $362.5 \mathrm{~mm}$. As shown in Fig. 12, the first three months of 1991 were more rainy than the first quarter of 1992. We found little seasonal change since precipitation varied drastically from year to year; nevertheless, June to November is the driest period.

\subsection{Photometric data: Atmospheric transparency}

The first results of daytime photometry at Oukaimeden site were published by Benkhaldoun et al. (1993).

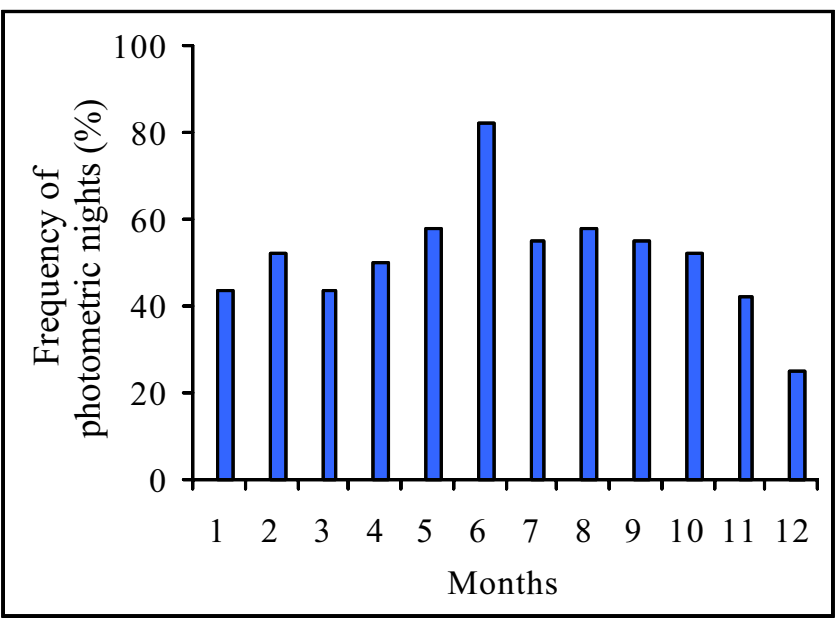

Fig. 14. Monthly variation of photometric nights at ORM

He found an extinction coefficient of $0.13 \mathrm{mag} /$ air mass and a fraction of clear weather of $65 \%$. Measurements of solar luminosity were performed simultaneously at three wavelengths $(550,589,700 \mathrm{~nm})$. The instrument used for those observations is a simple Flux Integration Photometer (FIP). For the description of this instrument and data reduction see Benkhaldoun (1993). The photometric quality of Oukaimeden is evaluated by using the least squares method applied to the data set. The atmospheric extinction coefficient is determined from the Bouguer straight line technique (Benkhaldoun 1994).

Using GONG data Hill et al. (1994a,b) found a slightly different result for the clear weather fraction statistics $(50 \%)$ and the extinction coefficient equals to $0.12 \mathrm{mag} / \mathrm{air}$ mass.

If the values found for the extinction are analogous (0.13 and 0.12), it is not the case for the clear time fraction $(65 \%$ and $50 \%)$. This difference is partly systematic explaining by the way of conception of the two instruments, the FIP with his system of diffuser measuring the totality of the light in source of sky whereas the GONG instrument measures the incidental direct radiance.

Indeed, during another observation period, (January 1992 - November 1992), we performed a cross calibration between the two instruments, which gave us a fraction of clear weather at Oukaimeden of $57 \%$ for FIP and $53 \%$ for GONG (Siher et al. 1996).

Another reason that lowers the fraction of clear weather issued by the GONG instrument lies in the frequency of the breakdowns. The most significant cause of breakdowns is due to power failures that occur during storms. The GONG instrument does not work for several days, when we generally experience clear and favorable weather. Meanwhile, during this time, the FIP instrument is working normally.

A battery gives an autonomy for a limited amount of time to GONG experiment, but it requires an human 
intervention to start again, after this time out. On the contrary, the FIP experiment automatically switches on after a breakdown. This affects the overall statistics as it has been shown by Benkhaldoun (1994). He demonstrates that, over three different periods (at the begining, in the middle and at the end of the measurement campaign), a decrease of $20 \%$ of breakdown periods results in a $10 \%$ increase of clear time fraction.

The 1997 IRIS results (Benkhaldoun et al. 1998) are in agreement with the first photometric data published by Benkhaldoun et al. (1993).

\section{ORM climatology based on CAMC database}

Previous research concerning meteorological parameters at ORM measured in different locations of the Observatory used data gathered from the telescopes as part as its routine operation. The wider and more systematic meteorological record comes however from the Carlsberg Automatic Meridian Circle Telescope $(\mathrm{CAMC})^{2}$. This telescope has been in continuous operation since 1984 in order to determine accurate star positions, as well as provide standard surface meteorological data to the astronomical community. From the monitoring of stars during the night extinction coefficients in the broad Johnson $V$ band are computed for every single night.

The CAMC data were taken with sensors attached to a mast installed near the telescope building and include measurements of air temperature, atmospheric pressure, wind speed and direction and relative humidity. The interior air temperature of the telescope building is also taken. The data set is completed with the values of nightly atmospheric extinction in addition to quality flags for each meteorological parameter.

The meteorological sensors were read automatically by an Olivetti desk-top computer and stored on disk. Readings were made at 5 minute intervals throughout the day and night, even during unfavourable astronomical conditions. No readings were available when the computer was down or a sensor became faulty. The atmospheric extinction $\left(A_{V}\right)$ is referred to the zenith (Carlsberg 1993).

The database includes more than ten years of measurements, ranging from 1984 to 1995 from which we excluded the volcanic eruption periods, before 1987 January and after 1991 April (Guerrero et al. 1998).

\footnotetext{
2 The Carlsberg Automatic Meridian Circle is operated by the Copenhagen University Observatory, The Institute of Astronomy (formerly by the Royal Greenwich Observatory) and the Real Instituto y Observatorio de la Armada en San Fernando, Cadiz.
}

\subsection{Air temperature}

The statistical results of the air temperature at CAMC site are presented in Figs. 2-4. In Fig. 2, we present the monthly variation of the mean, minimum and maximum temperature at ORM. The daily mean temperature is $8.37{ }^{\circ} \mathrm{C}\left(4.95{ }^{\circ} \mathrm{C}\right.$ in winter, $11.42{ }^{\circ} \mathrm{C}$ in summer $)$. The absolute minimum and maximum are $-9.4^{\circ} \mathrm{C}$ and $27^{\circ} \mathrm{C}$ respectively. The night-time and daytime mean temperature are $7.27{ }^{\circ} \mathrm{C}$ and $9.8^{\circ} \mathrm{C}$, respectively. In Fig. 3 we noticed a small variation of temperature during the day, and remarkable stability during the night-time.

Figure 4 shows the difference in temperature between two consecutive nights at ORM. We noticed a very small variation (in the mean curve), which confirms the high stability of the night-to-night temperature at ORM. The mean difference value is $1.6{ }^{\circ} \mathrm{C}$ which gives a dome and mirror seeing about 0.17 and 0.70 arcsec, respectively. The maximum difference value is $8.6^{\circ} \mathrm{C}$ recorded in November which might give rise to a dome and mirror seeing contribution about 1.32 and 5.29 arcsec, respectively.

\subsection{Atmospheric pressure}

Figure 13 shows the monthly variation of the atmospheric pressure. We observed that the pressure has low seasonal change and the mean pressure is 774.12 mbar higher than the theoretical pressure $88 \%$ of time. The theoretical value, calculated using a standard model, is equal to 760 mbar (Triplet \& Roche 1986). Therefore, ORM is dominated by high pressure, the signature of stable good weather. The absolute maximum is 784.47 mbar recorded in January and the absolute minimum is 751.4 mbar recorded in December.

We also noticed that during the summer, there is little prevailing high pressure and extreme values are close, as is to be expected from anticyclonic conditions.

\subsection{Relative humidity}

The variation of the monthly relative humidity show a seasonal effect (Fig. 5). The daily mean humidity is $40 \%$. The daytime and night-time mean humidity is $38 \%$ and $41 \%$, respectively. We noticed that the humid months are from October to March $(R H>40 \%)$, whereas the dry months is from April to September $(R H<40 \%)$. The percentage of time with humidity lower than $90 \%$ is $89 \%$ (Fig. 6), ( $45 \%$ occurring in winter, $55 \%$ occurring in summer).

Figure 7 shows the daily relative humidity at ORM. We noticed very little variation.

\subsection{Wind speed and direction}

Wind behaviour at various sites within the ORM has been studied by McInnes \& Walker (1974), Brandt \& Righini (1985) and Mahoney et al. (1998). 
Table 4. Night-time wind statistics. Percentage of time in sector (in\%) at ORM

\begin{tabular}{|c|c|c|c|c|c|c|c|c|c|c|c|c|c|c|c|c|c|}
\hline & $\mathrm{N}$ & NNE & $\mathrm{NE}$ & ENE & $\mathrm{E}$ & ESE & SE & SSE & S & SSW & SW & WSW & W & WNW & NW & NNW & all \\
\hline A & 4.3 & 3.7 & 4.3 & 3.3 & 2.3 & 2.3 & 4.4 & 2.9 & 2.1 & 1.9 & 1.6 & 1.3 & 1.3 & 1.7 & 1.9 & 2.2 & 41.5 \\
\hline B & 4.9 & 2.2 & 3.8 & 3.7 & 2 & 1.3 & 2.1 & 2.2 & 2.5 & 2.8 & 3.4 & 2.5 & 1.4 & 2 & 3.4 & 5.8 & 46 \\
\hline C & 1.3 & 0.2 & 0.3 & 0.6 & 0.4 & 0.2 & 0.3 & 0.3 & 0.8 & 1.4 & 2.1 & 0.9 & 0.3 & 0.3 & 0.7 & 2.4 & 12.5 \\
\hline & 10.5 & 6.1 & 8.4 & 7.6 & 4.7 & 3.8 & 6.8 & 5.4 & 5.4 & 6.1 & 7.1 & 4.7 & 3 & 4 & 6 & 10.4 & 100 \\
\hline
\end{tabular}

Table 5. Daytime wind statistics. Percentage of time in sector (in\%) at ORM

\begin{tabular}{|c|c|c|c|c|c|c|c|c|c|c|c|c|c|c|c|c|c|}
\hline & $\mathrm{N}$ & NNE & $\mathrm{NE}$ & ENE & $\mathrm{E}$ & ESE & $\overline{\mathrm{SE}}$ & SSE & $\bar{S}$ & SSW & SW & WSW & $\mathrm{W}$ & WNW & NW & NNW & all \\
\hline $\bar{A}$ & 6.7 & 4.6 & 3.8 & 2.5 & 1.4 & 1.3 & 2.6 & 4.5 & 3.7 & 2.3 & 2.1 & 2.2 & 1.9 & 2 & 2.6 & 3.6 & 47.8 \\
\hline B & 4.2 & 1.6 & 2.1 & 2.2 & 1.3 & 1 & 2 & 3.3 & 2.5 & 2.1 & 3.3 & 4.1 & 2.7 & 2.2 & 3.3 & 4.3 & 42.2 \\
\hline $\mathrm{C}$ & 0.8 & 0.1 & 0.3 & 0.3 & 0.2 & 0.2 & 0.3 & 0.3 & 0.6 & 0.9 & 1.7 & 1.5 & 0.6 & 0.3 & 0.6 & 1.3 & 10 \\
\hline & 11.7 & 6.3 & 6.2 & 5 & 2.9 & 2.5 & 4.9 & 8.1 & 6.8 & 5.3 & 7.1 & 7.8 & 5.2 & 4.5 & 6.5 & 9.2 & 100 \\
\hline
\end{tabular}

Our results for the CAMC site are shown in Tables 4 and 5 for night- and daytime, respectively, and in Figs. 8-11, which, respectively, show for the OUK and CAMC sites the night-time wind roses, the daytime wind roses, the monthly variation in wind speed and cumulative frequency plots of wind speed. There is a dramatic difference between the two sites.

The wind roses for the CAMC site are compiled from data taken from 1987 January to 1995 July. At night (Fig. 8), all directions are indicated, but with $\mathrm{N}$ and NNW being prevalent and with less conspicuous components from the NE and ENE. During the daytime, however, there is a significant change in the wind-direction pattern; the prevailing tendency is still from the $\mathrm{N}$ and NNE but there is more scatter in all directions with a pronounced increase in southerly (especially SSE) components. These results are in broad agreement with the findings of McInnes (1976) for the Fuente Nueva site, close to where the CAMC is situated (see his wind rose reproduced in Brandt \& Righini 1985). Note, however, that daytime measurements carried out by Brandt \& Wohl (1982, see their Fig. 9, bottom right) on the neighbouring slope of the Roque de los Muchachos show a slightly different wind pattern with a much more pronounced easterly component, which is absent in our Fig. 9 (right) for the CAMC site.

Our night-time results differ from those of Mahoney et al. (1998, see their Fig. 1), based on data gathered at the site selected for the spanish $10 \mathrm{~m}$ (GTC) telescope (Muñoz-Tuñón et al. 1998). Mahoney et al. find a NE-ENE prevailing wind direction for GTC site (located below the Roque de los Muchachos on the same slope). They attribute this difference in wind pattern to local orographical influences. The NE-SE axis that is so prominent in their day- and night-time wind roses runs parallel to the local orographical contours of the GTC site; in other words, the prevailing free-air wind (coming from the NW as it reaches the Canaries) is diverted by the obstacle presented by the local orography. A plausible explanation for the discrepant behaviour of the wind at the Fuente Nueva and GTC sites could be that the contours at the former site run E-W while the site itself is situated immediately above a deep gorge running N-S, an orographical combination conducive to diverting the prevailing free-air $\mathrm{NW}$ wind into the $\mathrm{N}$ direction.

Figure 10 shows that mean wind speed varies very little. In Fig. 11, we observe that the wind speed at the site is generally low, with strong winds blowing for $0.01 \%$ of the time. As we see for April, the mean wind velocity is $2.8 \mathrm{~m} \mathrm{~s}^{-1}$, the absolute maximum is $23 \mathrm{~m} \mathrm{~s}^{-1}$.

\subsection{Photometric data: Atmospheric extinction}

The atmospheric extinction in the Canary Islands has been studied by Murdin (1985), Stickland et al. (1987), Whittet et al. (1987) Jimenez, González Jorge (1998) and Guerrero et al. (1998, 2000). To characterize ORM's site properties, Guerrero et al. ignored the external effect of eruptions of El Chichón and Mt. Pinatubo their extinction estimates. They found a modal value of $0.11 \mathrm{mag} /$ airmass and their database included over 1269 extinction values. The extinction increases in the summer months (especially July and August) due to occasional irruptions of dust in suspension brought to the observatories by southerly winds from the Sahara Desert. For a more complete study of dust properties on the atmospheric extinction see Jimenez et al. (1998).

We have also studied the photometric conditions at the ORM site. CAMC data from 1984 May to 1998 May (2931 extinction values) show that the extinction varies between 0.08 to $0.98 \mathrm{mag} /$ airmass, the modal value being $0.12 \mathrm{mag} /$ airmass. Figure 14 records the frequency of photometric nights (for a definition of photometric nights see Murdin 1985). These occur with a mean annual frequency of $51 \%$ ( $40 \%$ in the winter and $60 \%$ in the summer). The maximum frequency of occurrence of photometric 
nights is $82 \%$ in June and the minimum is $25 \%$ in December.

The percentage of photometric nights is higher in summer, than in winter although during the months of July, August and September the sky transparency over the ORM site is occasionally affected by irruptions of airborne dust from the Sahara desert which is carried up to $3 \mathrm{~km}$ in altitude by southern winds which blow over the Atlantic Ocean.

\section{Comparison Oukaimeden/ORM}

Table 6 presents both meteorological and photometric characteristics of the two sites. Both sites are in a subtropical zone of high pressure. Therefore, despite the difference in orographical surroundings, they provide similiar observational conditions. Also, at both sites, the temperature inversion layer is usually quite low. When this condition occurs, we occasionally notice the presence of a cloud layer below the site, there being a direct correlation between image quality and cloud height (Muñoz-Tuñón et al. 1997).

The mean temperature difference between the two sites where the altitude difference is approximately $374 \mathrm{~m}$, is $1.9^{\circ} \mathrm{C}$, which is very close to the value of $1.8^{\circ} \mathrm{C}$ predicted by Triplet \& Roche (1986).

Figure 3 shows that the temperature at both sites decreases very slowly during the night, (by less than one degree), due to thermal cooling. The low surface layer contributes to excellent observing conditions at the ORM, already measured by Vernin \& Muñoz-Tuñón (1994), leading us to predict the same excellent conditions at Oukaimeden.

The variation in temperature between two successive nights is approximately the same at both sites, i.e. $\sim 1.6^{\circ} \mathrm{C}$.

In Fig. 7, we show that relative humidity is higher during the winter months at Oukaimeden, and that the monthly mean values can differ by more than a few percentage points between the two sites. Thus the relative humidity variation at night is much higher at Oukaimeden.

We can observe a correlation between temperature and relative humidity: the warmest summer months (typically June and August) are marked by extremely low relative humidity, whereas the coldest winter months (from October to April) have the highest relative humidity and lowest temperature.

Wind statistics show the prevalence of low wind speeds at both sites. The nocturnal data show that mean wind velocity is less than $3 \mathrm{~m} \mathrm{~s}^{-1}$, so that the OUK site may be considered to be suitable for astronomy. The nighttime and daytime wind speeds at both sites are lower than $15 \mathrm{~m} \mathrm{~s}^{-1}$ for $99 \%$ of the time.

There is a change in the wind roses for day- and nighttime at each site and no obvious comparison can be made.
Due to the presence of a semi-permanent anticyclone, which follows the change in declination of the Sun (Font Tullot 1956), the trade winds are predominant in the Canary Islands. In the summer, when the anticyclone extends to Morocco, the weather becomes dry and hot. However, low pressure zones in the southern part of the country often cause local storms. In the winter the Azores anticyclone moves towards south. At the same time, depressions are formed in the Northern Atlantic and moves eastwards. The weather in these conditions becomes humid and cold (Benkhaldoun 1994).

Because no sky quality measurements at Oukaimeden were available, we tried to compare the extinction trend at both sites by using daytime data at Oukaimeden and night-time data at the ORM.

We selected a range of CAMC data (1988 October to 1991 April) that coincided with measurements made at Oukaimeden. We compared 620 extinction values at Oukaimeden and 511 at the ORM (Fig. 15), during a period which was not affected by volcanic eruptions (Guerrero et al. 1998). The mode and median values of the atmospheric extinction coefficient at ORM are equal to $0.12 \mathrm{mag} /$ airmass. These results confirm the excellent sky transparency above the ORM and converge toward those found by Guerrero et al. (cf. Sect. 4.5). At Oukaimeden the mode value is $0.10 \mathrm{mag} /$ airmass and the median value is $0.12 \mathrm{mag} /$ air mass, which equal Hill's findings (1994a,b).

We can see a clear seasonal effect at both sites with an increase in the extinction coefficient in the summer. The occasional irruption of spectrally grey Saharan dust (see Stickland et al. 1987; Whittet et al. 1987) leads to a temporary increase in the extinction coefficient. This effect is moderate at Oukaimeden due to the presence of the intervening Atlas Mountains, whose altitude exceeds $4000 \mathrm{~m}$, which shield the site from dust and wind.

Figure 16 shows a subset of days for which simultaneous measurements were made. The data are reduced to 360 extinction values and there is no correlation between the two sites. The median is about $0.12 \mathrm{mag} /$ airmass at both sites, the mode is $0.12 \mathrm{mag} /$ airmass at ORM and $0.10 \mathrm{mag} /$ airmass at Oukaimeden. Even if only $360 \mathrm{ex}-$ tinctions are analysed, daytime condition at Oukaimeden are comparable to night-time conditions at ORM.

These values compare favourably to previous observations made at other sites, e.g. $0.15 \mathrm{mag} / \mathrm{air}$ mass for Mt. Wilson (Schmidt-Kaler 1982) and $0.124 \mathrm{mag} /$ air mass for La Silla (Rufener 1986).

\section{Conclusion}

A site evaluation program was planned to provide some basic statistics on Oukaimeden surface meteorology and atmospheric extinction in order to promote the installation of a permanent observatory. 
Table 6. Local characteristics of the Oukaimeden and CAMC site

\begin{tabular}{|c|c|c|c|}
\hline & & ORM & OUKAIMEDEN \\
\hline GEOGRAPHY & UNIT & & \\
\hline Longitude & deg, min, sec & $175257 \mathrm{~W}$ & $75252 \mathrm{~W}$ \\
\hline Latitude & deg, min, sec & $284536 \mathrm{~N}$ & $311232 \mathrm{~N}$ \\
\hline distance to nearest city (time) & $\mathrm{km}(\mathrm{h})$ & $(2 \mathrm{~h})$ & $80(1 \mathrm{~h})$ \\
\hline Altitude $\mathrm{m}$ above sea level & $(\mathrm{m})$ & 2326 & 2700 \\
\hline Shape & & ridge of Caldera & peak \\
\hline Distance to coast & $\mathrm{km}$ & $10-12$ & 100 \\
\hline \multicolumn{4}{|l|}{ LOCAL CLIMATOLOGY } \\
\hline \multirow[t]{5}{*}{ Temperature } & mean value $\left({ }^{\circ} \mathrm{C}\right)$ & 8.37 & 6.47 \\
\hline & winter/summer & $4.95 / 11.42$ & $1.96 / 12.36$ \\
\hline & $\min , \max$ & $-9.4,27$ & $-12.9,25.6$ \\
\hline & $<\left|T_{j}-T_{j+1}\right|>_{\text {moy }}$ & 1.6 & 1.72 \\
\hline & $<\left|T_{j}-T_{j+1}\right|>_{\max }$ & 8.6 & 9 \\
\hline \multirow[t]{3}{*}{ Relative humidity } & mean value $(\%)$ & 40 & 45 \\
\hline & winter/summer & $47.99 / 31.56$ & $47.70 / 41.25$ \\
\hline & prob. $(R H<90 \%)(\%)$ & 89 & 92 \\
\hline \multirow[t]{5}{*}{ Pressure } & mean value $(\mathrm{hPa})$ & 774.12 & \\
\hline & $\min , \max$ & $751.4,784.47$ & \\
\hline & theoretical value $P_{\mathrm{t}}$ & 760 & 724 \\
\hline & $\%$ of time with $P>P_{\mathrm{t}}$ & 88 & \\
\hline & winter/summer & $773.26 / 774.91$ & \\
\hline \multirow[t]{6}{*}{ Wind } & mean value velocity $\left(\mathrm{m} \mathrm{s}^{-1}\right)$ & 2.8 & 2.63 \\
\hline & $\max$ & 23 & 50 \\
\hline & velocity lower than $5 \mathrm{~m} \mathrm{~s}^{-1}(\%)$ & 82 & 89.22 \\
\hline & velocity lower than $15 \mathrm{~m} \mathrm{~s}^{-1}(\%)$ & 99.99 & 99.84 \\
\hline & night-time predominant wind (\%) & $\mathrm{N}(11.63)$ & $\mathrm{SE}(21.80)$ \\
\hline & daytime predominant wind $(\%)$ & $\mathrm{N}(10.45)$ & WNW $(13.16)$ \\
\hline Precipitation & $(\mathrm{mm})$ & & 362.5 \\
\hline \multirow[t]{3}{*}{ Photometry } & atmos. ext. coef. (mag/airmass) & 0.11 (night-time data) & 0.12 (daytime data) \\
\hline & frequency of photometric nights $(\%)$ & 51 & \\
\hline & fraction of clear weather $(\%)$ & & 65 \\
\hline
\end{tabular}
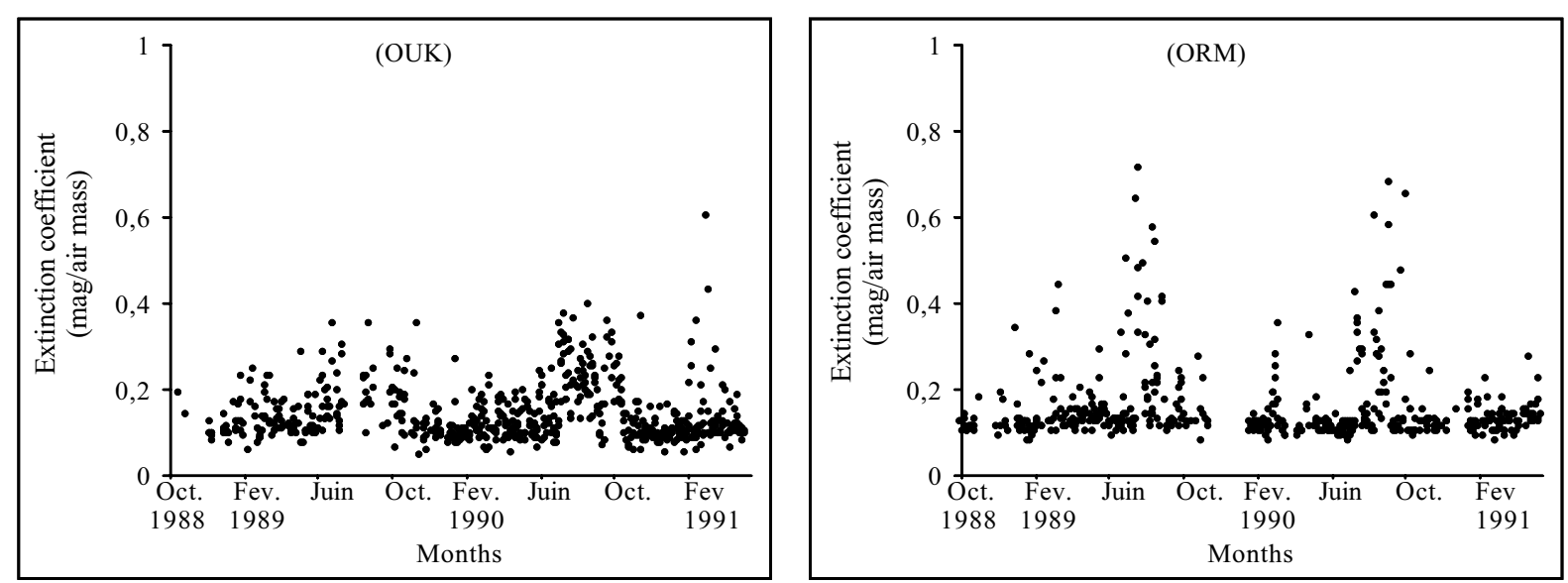

Fig. 15. Extinction measurements from 01/10/1988 to 30/04/1991 at Oukaimeden (left) and ORM (right). The increase in atmospheric extinction appears clearly in summer 1989 and 1990 at both sites 


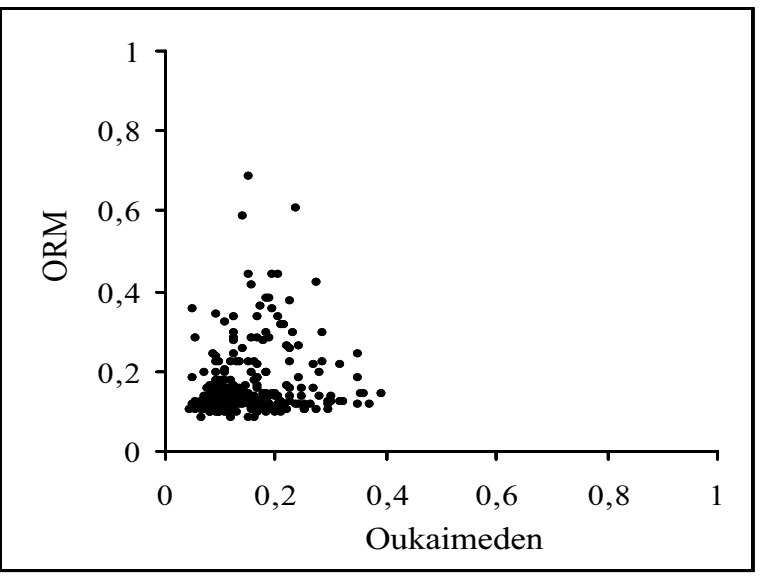

Fig. 16. Simultaneous extinction measurements at both sites from $01 / 10 / 1988$ to $30 / 04 / 1991$. The Oukaimeden data is displayed in $X$ axis and ORM data in $Y$ axis

The results of this study support the following conclusions.

The mean night-time temperature is moderate all year round. Extreme temperatures rarely seem to occur and the stability of the night-time temperature is consistent. The wind should not constitute a major problem for astronomical observations since the average wind speed is normally low. The predominant wind direction is from the SE during the night and from WNW during the day. This is not harmful, given the position of the site with respect to the high Atlas Mountains in SE and SW directions. Local humidity is almost always modest. The atmospheric extinction is comparable to the best sites in the world.

The results of analysis from the CAMC data confirm those of the numerous studies made in the past at various sites within the ORM, showing potentially optimum conditions for astronomical observations.

The comparison of both sites and analysis of the measured values allow us to conclude that Oukaimeden is an excellent sites in the northern hemisphere; being a suitable candidate for the installation of new astronomical equipment.

Our research at Oukaimeden needs to continue, particularly with regard to image quality. Atmospheric extinction results reveal the high quality of transparency at both sites, and the frequency of days and nights during which photometric measurements are feasible.

Acknowledgements. This work has been made possible thanks to the Franco-Moroccan cooperation (050/STU/97). We are very grateful to Claus Fabricius for providing the CAMC database and to the Moroccan National Direction of Meteorology. We also want to give our thanks to Terence Mahoney for his comments and careful reading of the manuscript.

\section{References}

Ardeberg A., 1983, Proceeding of the ESO Workshop on Site Testing for Future Large Telescope, La Silla, 4-6 October

Benkhaldoun Z., Kadiri S., Lazrek M., Vernin J., 1993, Exp. Astron. 2, 345-356

Benkhaldoun Z., 1994, thèse d'État, Cadi Ayyad University, Marrakech, Morocco

Benkhaldoun Z., Siher E., 1998, Soho/Gong98 Workshop, Boston, U.S.A., p. 109-113

Brandt P.N., Wohl H., 1982, A\&A 109, 77

Brandt P.N., Righini A., 1985, VA 28, 437

Cardelli J.A., Ackerman T.P., 1983, PASP 95, 451

Carlsberg Meridian Catalogue, 1993, Copenhagen University Observatory, Royal Greenwich Observatory, and Real institutes y Observatorio de la Armada de San Fernando

Guerrero M.A., et al., 1998, Muñoz-Tuñón C. (ed.), New Astron. Rev. 42, 529

Guerrero M.A., Fuensalida J.J, Garcia López R.J., Corradi R.L.M., Jiménez A., Alonso A., Rodriguez Espinosa J.M., Centurión M., Prada F., 2000 (submitted to PASP)

Helmer L., Morrison L.V., 1985, Vistas Astron. 28, 505

Hill F., 1994a, Solar Phys. 152, 321

Hill F., 1994b, Solar Phys. 152, 351

Jabiri A., 1994, thèse, Cadi Ayyad University, Marrakech, Morocco

Jabiri A., Benkhaldoun Z., Siher L., 1995, VII Iris Workshop, Univ. Cadi Ayyad, Marrakech, Morocco

Jiménez A., Gonzales Jorge H., Rabello-Soares M.C., 1998, A\&AS 129, 1

Kadiri S., 1983, thèse de Doctorat, University of Nice

Mahoney T., Muñoz-Tuñón C., Varela A.M., 1998, MuñozTuñón C. (ed.), New Astron. Rev. 42, 417-424

Mc Innes B., Walker M.F., 1974, MNRAS 86, 529

Mc Innes B., 1981, Q.IL.R. Astr. Soc. 22, 266-271

Muñoz-Tuñón C., Varela, A.M., Mahoney, T., 1998, MuñozTuñón C. (ed.), New Astron. Rev. 42, 409

Muñoz-Tuñón C., Vernin J., Varela A.M., 1997, A\&A 125, 183-193

Murdin P., 1985, Vistas Astron. 28, 449

Racine R., Salmon D., Cowley D., Sovka J., 1991, PASP 103, $1020-1032$

Rufener F., 1986, A\&A 165, 275-286

Sarazin M., 1995, Technical note on Site Quality Assessment: Observatorio del Roque de los Muchachos, October 26

Schmidt-Kaler Th., 1982, NS Vol. 2b, Schaifers K., Voigt H.H. (eds.). New York, Springer

Siher E., Benkhaldoun Z., Jabiri A., 1996, VIII Iris Workshop, Univ. Nice, France

Stickland D.J., Lloyd C., Pike C.D., Walker E.N., 1987, Observatory 107, 74

Triplet J.P., Roche G., 1986, $3^{\mathrm{e}}$ edition (reprint 1996).

Vernin J., Muñoz-Tuñón C., 1992, A\&A 257, 811

Vernin J., Muñoz-Tuñón C., 1994, A\&A 284, 311

Whittet D.C.B., Bode M.F., Murdin P., 1987, Vistas Astron. 30, 135-144 